\title{
Improved Traffic Prediction by Applying KNN and Euclidean Distance ARIMA (Ke-Arima) Approach
}

\author{
Priyanka Rani \\ Computer Science \& Engineering \\ I.K.G Punjab Technical University \\ Kapurthala- Jalandhar Highway
}

\begin{abstract}
In the modern era, the road infrastructure failed to cope up with the exponential increase of road traffic. There is a thrust to find a smarter way to deal with such transportation system. Intelligent Transport System is at the forefront edge of this, one of the points is exact and hassle-free forecasts that guarantee smooth and bother free driving and authoritative experience. In such manner, Intelligent Transport System (ITS) being looked into for quite a few years and furthermore a field of consistent growth of works and advancement after some time, there is a wealth of writing on traffic expectation. Traffic datasets generated through the application of IOT are operated upon by the existing techniques. Traffic flow analysis is conducted to tackle the issues of traffic forecasting. This paper presents a systematic analysis of previous aggregate work on traffic prediction, highlight the marked changes and presents future directions for research work.
\end{abstract}

\section{Keywords}

Traffic prediction, Traffic Dataset, Internet of Things (IOT), Traffic flow, traffic forecasting, Intelligent Transport System (ITS).

\section{INTRODUCTION}

Intelligent transportation system is a technique or an application in electronic or non-electronic forms for producing information through advanced sensors, computers and communication technology that improve the process of traffic forecasting. ITS is wide field providing assistance in the field of driver assistance, inter-vehicle communication, air traffic control, road sign prediction, number plate detection, congestion control, dynamic routing etc. It's caters to the multidimensional needs of traffic management overlapped with number plate detection and road traffic signal prediction. Most of the issues of traffic prediction are caused due to existing infrastructure; however, some of the issues are also caused by poor management of traffic flow and congestion control. ITS tackles the issue of poor management of traffic flow by the use of accurate traffic monitoring and control strategies. The distributed and shared judgment and care management have been remolded an open issue at all levels of traffic forecasting systems. For the estimation of traffic prediction, it requires the information that is simple and diverse from the sensors and skills.

To work efficiently there should be an ITS software system in this environment. But this system also requires credible and timely information to ensure that software can work securely and produce results within specified time. Computer systems make the interaction between human and computational devices very natural so that users can get desired data in a transparent manner. The newly introduced gadgets like mobiles, PDAs, laptops etc. make every information available anywhere at any time.
By using ITS, interactive feedback loops and video games, we can analyze the traffic-related behavior changes that may occur. ITS is associated with many applications and in longterm it is viable to get feasible into larger frameworks in health care. According to researchers it is suggested that use of ITS and emergence in technology is efficient enough to aware users about the current traffic and provide preventive measures. The ITS also enable the user for behavior change. Distinct elements of ITS are the enhancement in decision making an object-oriented. Diverting the traffic greatly depend upon the awareness of driver which will be accomplished by the use of ITS. Routing adherence is greatly impacted by this mechanism. With the help of transportation system, drivers can analyze his behavior and prepare him for taking appropriate action

\section{LITERATURE SURVEY}

To tackle the requirements of a systematic review, background analysis is conducted. The background analysis presents the existing techniques that are comprehensively used to predict road traffic.

[1] This paper depicted our examination encounters of building a keen framework to screen and control street traffic in a Nigerian city. A half and half approach got by the intersection of the Structured Systems Analysis and Design Methodology (SSADM) and the Fuzzy-Logic based Design Methodology was conveyed to create and actualize the framework. Issues were related to the present traffic control framework at the ' + ' intersections and this required the plan and usage of another framework to take care of the issues. The subsequent fluffy logic-based framework for activity control was recreated and tried utilizing a prominent crossing point in a Nigerian city; infamous for extreme activity logjam. The new framework dispensed with a portion of the issues distinguished in the current activity checking and control frameworks.

[2]Traffic flag controller is playing increasingly and more critical parts in present-day administration and control of urban traffic. This paper introduces a shrewd traffic flag controller in light of multi-microcomputer innovation. The architecture and crucial elements of the clever traffic flag controller $U$ initially presented in detail, at that point the human-PC interface in light of visual innovation intended for the controller is figured, and lastly an application case by and by is talked about.

[3]Propelled activity data benefit framework not just give opportune and precise traffic data for activity administration workforce who can adequately adjust the traffic administration control framework to an assortment of traffic conditions and street arrange limit, yet in addition, help street clients, viably staying away from roads turned parking lots, diminishing auto collisions. Notwithstanding, the existing dynamic activity data is discharged for the general group of 
onlookers. On the off chance that the majority of the drivers utilize the dynamic traffic data to design ongoing travel courses, at that point the in general activity framework might be bothered generally, and another road turned parking lot appear in the meantime maintaining a strategic distance from the current activity stick. In light of the GIS spatial information demonstrate and the hypothesis of multi-operator, we ponder a dynamic activity data administrations innovation in view of collective multi-specialist techniques all together to show signs of improvement travel way through upgrading the communication, what's more, coordinated effort between the data suppliers and voyagers. At that point, the test model framework is outlined, what's more, created in view of the swarming stage and Java language, and some analysis data is produced by the prototype system.

[4] Dealing with the expanding activity is a major issue everywhere throughout the world. Wise Transportation System (ITS) gives an answer for these issues with the assistance of new advancements. ITS is an incorporated framework that executes an expansive scope of correspondence, control, vehicle detecting and hardware advances to take care of and deal with the traffic issues. ITS is being utilized as a part of the created nations since past two decades, however, it is as yet another idea when creating nations like India, Brazil, China, South Africa and so on is concerned. In the present examination, we have considered four noteworthy parts of the ITS i.e., Advanced Traveler Data System (ATIS), Advanced Traffic Management System (ATMS), Advanced Public Transportation System (APTS), and Emergency Management System (EMS). The target of the paper is to ponder different ITS engineering and model and audit such models to get top to bottom of their design. Subsequently, engineering and created models throughout the times of four noteworthy branches of ITS have been inspected here to make an examination investigation of various models that have been produced by the scientists in their examinations. It will prompt the holes in the information which can be additionally considered. The paper features the conclusions extricated from the investigations of various frameworks and furthermore gives what's to come scope in the field of ITS to make it more easy to use and open.

[5] As of late notoriety of private autos is getting urban activity more swarmed. As result traffic is getting to be plainly one of the vital issues in huge urban areas everywhere throughout the world. A portion of the activity concerns is clogs and mischance which have caused a colossal exercise in futility, property harm, and ecological contamination. This exploration paper introduces a novel smart activity organization framework, in view of an Internet of Things, which is included by ease, high adaptability, high similarity, simple to redesign, to supplant conventional traffic administration framework and the proposed framework can enhance street activity hugely. The Internet of Things depends on the Internet, organize remote detecting and discovery advances to understand the canny acknowledgment of the labeled activity protest, following, observing, overseeing and handled naturally. The paper proposes a design that coordinates web of things with operator innovation into a solitary stage where the specialist innovation handles successful correspondence and interfaces among countless exceptionally dispersed and decentralized gadgets inside the IOT. The design presents the utilization of a dynamic radiorecurrence distinguishing proof (RFID), remote sensor advances, question specially appointed systems administration, and Internet-based data frameworks in which labeled activity items can be consequently spoken to, followed, and questioned over a system. This examination shows a review of a structure conveyed traffic reproduction display inside NetLogo, an operator based condition, for IOT activity checking framework utilizing versatile specialist innovation.

[6]This paper incorporates the plan and usage of a clever and robotized activity control framework which takes points of interest of PC vision and picture handling systems. Alongside regular PC vision strategies; this paper presents two new techniques which have low preparing cost. One of the techniques has been developed with the assistance of equipment what's more; the other one is outlined without equipment bolster. This is a finishing activity administration framework which has possessed the capacity to decrease roads turned parking lots and clog on re-enacted condition. It distinguishes the number of vehicles on every street and relying upon the vehicles stack on every street, this framework allows the improved sum of holding up time (red flag light) and running time (green flag light). This framework is a completely robotized framework that can supplant the regular pre-decided settled time-based activity framework with a progressively oversaw activity framework. It can likewise distinguish vehicle condition on street and autochange the framework as indicated by the changing street conditions which make the framework insightful. The composed framework can help to tackle traffic issues in occupied urban communities to an awesome degree by sparing a lot of worker hours that get lost attending to stuck streets. This examination concentrates on factors, ease picture preparing an activity stack adjusting.

[7] As indicated by city open travel issue trademark, the fundamental body of a paper has been submitted and has worked out one sort of in view of the Internet of things outline intelligent transportation framework. That framework gathers information by vehicle terminal and transfers information to the server through the system and makes information obvious to the purchaser passing an algorithm in the server. One viewpoint, the customer may ask about open travel vehicle data by Web. On another viewpoint, the shopper can know open travel vehicle data by station terminal. The investigations have tried that the intelligent transportation framework can offer open travel vehicle data to numerous shoppers with helpful way along these lines this framework can take care of the city mass travel issue.

[8] This paper concentrated on the fundamental structure of canny urban Traffic Management System Based on Cloud Figuring and Internet of Things, proposed the design of canny urban Traffic Management System Based on Distributed computing and Internet of Things. The paper made a profound research on the data observing in light of Internet of things, estimation and the shrewd displaying segments, what's more, learning coordinating segment. Mass estimation was acknowledged by the utilization of the distributed computing stage. The framework generally understands the shrewd observing what's more, administration of urban traffic and understands the reason for the keen dig of urban traffic.Traffic management with the implication of sensors is complex and required accuracy. Techniques devised so far still requires further enhancements for increasing accuracy of prediction. Next section presents problem definition giving parameters which can be further enhanced.

\section{GAPS IN LITERATURE}

Analysis of literature indicates that dataset used is offline and is not derived with the application of IOT. Sensor data 
utilization within traffic related application is the prime cause of interest. Accurate prediction related to traffic to drivers involved along with direction sensing is missing in existing literature. Advanced application framework construction for traffic prediction is the solution to the problem.

\subsection{Comparision Table}

The comparison of various techniques that can be used to predict traffic is listed as under:-

Table 1: Comparison of various techniques that can be used to predict traffic

\begin{tabular}{|c|c|c|c|c|c|}
\hline Title & Technique & $\begin{array}{l}\text { Datas } \\
\text { ets }\end{array}$ & $\begin{array}{l}\text { Param } \\
\text { eters }\end{array}$ & Merit & $\begin{array}{l}\text { Deme } \\
\text { rit }\end{array}$ \\
\hline $\begin{array}{l}\text { A } \\
\text { Consumer } \\
\text { Transceiv } \\
\text { er for } \\
\text { Long- } \\
\text { Range } \\
\text { IOT } \\
\text { Communi } \\
\text { cations in } \\
\text { Emergenc } \\
\text { y } \\
\text { Environm } \\
\text { ents[12] }\end{array}$ & \begin{tabular}{|l} 
IEEE80 \\
2.11ah \\
Wi-Fi \\
protocol \\
, Time \\
Domain \\
Least \\
Square( \\
TDLS)
\end{tabular} & -------- & $\begin{array}{l}\text { Packet } \\
\text { Error } \\
\text { Rate(P } \\
\text { ER), } \\
\text { MSE }\end{array}$ & $\begin{array}{l}\text { Increa } \\
\text { sed } \\
\text { range } \\
\text { of } \\
\text { servic } \\
\text { e }\end{array}$ & $\begin{array}{l}\text { Time } \\
\text { of } \\
\text { execut } \\
\text { ion is } \\
\text { substa } \\
\text { ntially } \\
\text { high }\end{array}$ \\
\hline $\begin{array}{l}\text { The } \\
\text { advantage } \\
\mathrm{s} \text { of IOT } \\
\text { and Cloud } \\
\text { applied to } \\
\text { Smart } \\
\text { Cities[13] }\end{array}$ & $\begin{array}{l}\text { ClouT } \\
\text { architec } \\
\text { ture } \\
\text { which is } \\
\text { the } \\
\text { combin } \\
\text { ation of } \\
\text { cloud } \\
\text { and IOT } \\
\text { is } \\
\text { discusse } \\
\text { d }\end{array}$ & -------- & ------- & $\begin{array}{l}\text { Sensiti } \\
\text { zation, } \\
\text { Actual } \\
\text { isation } \\
\text { layer } \\
\text { along } \\
\text { with } \\
\text { IOT } \\
\text { have } \\
\text { been } \\
\text { added } \\
\text { in } \\
\text { CIaaS } \\
\text { layer } \\
\text { to } \\
\text { extract } \\
\text { data } \\
\text { out of } \\
\text { API's }\end{array}$ & $\begin{array}{l}\text { CSaaS } \\
\text { layer } \\
\text { is still } \\
\text { not } \\
\text { compl } \\
\text { etely } \\
\text { define } \\
\text { d. }\end{array}$ \\
\hline $\begin{array}{l}\text { Short- } \\
\text { term } \\
\text { traffic } \\
\text { flow } \\
\text { prediction } \\
\text { using } \\
\text { seasonal } \\
\text { ARIMA } \\
\text { model } \\
\text { with } \\
\text { limited } \\
\text { input } \\
\text { data[14] }\end{array}$ & $\begin{array}{l}\text { SARIM } \\
\text { A }\end{array}$ & $\begin{array}{l}\text { A 3- } \\
\text { Lane } \\
\text { roadw } \\
\text { ay in } \\
\text { Chenn } \\
\text { ai, } \\
\text { India }\end{array}$ & $\begin{array}{l}\text { A flow } \\
\text { of } \\
\text { vehicle } \\
\text { s' }^{\prime} \\
\text { accurac } \\
\text { y } \\
\text { through } \\
\text { MAPE }\end{array}$ & $\begin{array}{l}\text { More } \\
\text { accura } \\
\text { te } \\
\text { results } \\
\text { even } \\
\text { with } \\
\text { data } \\
\text { shorta } \\
\text { ge }\end{array}$ & $\begin{array}{l}\text { More } \\
\text { time } \\
\text { for } \\
\text { compu } \\
\text { tations }\end{array}$ \\
\hline $\begin{array}{l}\text { Smart } \\
\text { Disease } \\
\text { Surveillan } \\
\text { ce Based } \\
\text { on }\end{array}$ & $\begin{array}{l}\text { IOT in } \\
\text { the field } \\
\text { of } \\
\text { healthca } \\
\text { re }\end{array}$ & $\begin{array}{l}\text { Centra } \\
1 \\
\text { Health } \\
\text { Minist } \\
\text { ry }\end{array}$ & $\begin{array}{l}\text { Predicti } \\
\text { on } \\
\text { accurac } \\
\text { y }\end{array}$ & $\begin{array}{l}\text { Fast } \\
\text { predic } \\
\text { tion of } \\
\text { patter } \\
\text { ns of }\end{array}$ & $\begin{array}{l}\text { Inadeq } \\
\text { uate } \\
\text { data } \\
\text { manag } \\
\text { ers, }\end{array}$ \\
\hline
\end{tabular}

\begin{tabular}{|c|c|c|c|c|c|}
\hline $\begin{array}{l}\begin{array}{l}\text { Internet } \\
\text { of Things } \\
\text { (IOT) }\end{array} \\
{[15]}\end{array}$ & & & & $\begin{array}{l}\text { diseas } \\
\text { e, help } \\
\text { to take } \\
\text { measu } \\
\text { res on } \\
\text { time }\end{array}$ & $\begin{array}{l}\text { low } \\
\text { budget } \\
\text {, lack } \\
\text { of } \\
\text { techni } \\
\text { cal } \\
\text { adviso } \\
\text { ry } \\
\text { group }\end{array}$ \\
\hline $\begin{array}{l}\text { Optimisin } \\
\mathrm{g} \text { Power } \\
\text { Consumpt } \\
\text { ion of } \\
\text { Wi-Fi } \\
\text { inbuilt } \\
\text { IOT } \\
\text { Devices[1 } \\
\text { 6] }\end{array}$ & $\begin{array}{l}\text { Reduce } \\
\text { power } \\
\text { consum } \\
\text { ption of } \\
\text { Wi-Fi } \\
\text { enabled } \\
\text { devices }\end{array}$ & ------- & $\begin{array}{l}\text { The } \\
\text { power } \\
\text { consum } \\
\text { ption } \\
\text { of } \\
\text { various } \\
\text { process } \\
\text { ors }\end{array}$ & $\begin{array}{l}\text { Wi-Fi } \\
\text { is } \\
\text { better } \\
\text { than } \\
\text { other } \\
\text { techno } \\
\text { logies } \\
\text { in } \\
\text { terms } \\
\text { of } \\
\text { range } \\
\text { and } \\
\text { securit } \\
\text { y }\end{array}$ & $\begin{array}{l}\text { No } \\
\text { param } \\
\text { eters } \\
\text { enhan } \\
\text { cemen } \\
\text { ts are } \\
\text { sugges } \\
\text { ted }\end{array}$ \\
\hline $\begin{array}{l}\text { Energy- } \\
\text { Efficient } \\
\text { Location } \\
\text { and } \\
\text { Activity- } \\
\text { Aware } \\
\text { On- } \\
\text { Demand } \\
\text { Mobile } \\
\text { Distribute } \\
\text { d Sensing } \\
\text { Platform } \\
\text { for } \\
\text { Sensing } \\
\text { as } \\
\text { Service in } \\
\text { IOT } \\
\text { Clouds[1 } \\
\text { 7] }\end{array}$ & $\begin{array}{l}\text { C- } \\
\text { MOSD } \\
\text { EN } \\
\text { platfor } \\
\text { m }\end{array}$ & $\begin{array}{l}\text { Conte } \\
\text { xt, } \\
\text { activit } \\
\text { y, and } \\
\text { locatio } \\
\text { n- } \\
\text { aware } \\
\text { modul } \\
\text { e(Both } \\
\text { real } \\
\text { world } \\
\text { and } \\
\text { simula } \\
\text { ted } \\
\text { lab- } \\
\text { based } \\
\text { data } \\
\text { were } \\
\text { focuse } \\
\text { d on) }\end{array}$ & $\begin{array}{l}\text { Energy } \\
\text { Storage } \\
\text { Comm } \\
\text { unicati } \\
\text { on }\end{array}$ & $\begin{array}{l}\text { Sensor } \\
\mathrm{s} \\
\text { energy } \\
\text { is } \\
\text { conser } \\
\text { ved } \\
\text { and } \\
\text { increa } \\
\text { ses the } \\
\text { lifetim } \\
\text { e of } \\
\text { the } \\
\text { netwo } \\
\text { rk }\end{array}$ & $\begin{array}{l}\text { No } \\
\text { focus } \\
\text { on } \\
\text { privac } \\
\text { y } \\
\text { preser } \\
\text { vation } \\
\text { techni } \\
\text { que. }\end{array}$ \\
\hline $\begin{array}{l}\text { Internet } \\
\text { of Things: } \\
\text { Remote } \\
\text { Patient } \\
\text { Monitorin } \\
\text { g Using } \\
\text { Web } \\
\text { Services } \\
\text { and Cloud } \\
\text { Computin } \\
\text { g[18] }\end{array}$ & $\begin{array}{l}\text { Android } \\
\text { app is } \\
\text { framed } \\
\text { which } \\
\text { takes } \\
\text { data } \\
\text { from } \\
\text { IOIO- } \\
\text { OTG } \\
\text { board. } \\
\text { The } \\
\text { binary } \\
\text { file is } \\
\text { uploade } \\
\text { d on } \\
\text { cloud } \\
\text { and } \\
\text { process } \\
\text { ed using } \\
\text { MATL } \\
\text { AB }\end{array}$ & $\begin{array}{l}\text { Bio- } \\
\text { medic } \\
\text { al data } \\
\text { like } \\
\text { temper } \\
\text { ature, } \\
\text { pulse, } \\
\text { blood } \\
\text { pressu } \\
\text { re etc. }\end{array}$ & $\begin{array}{l}\text { Portabi } \\
\text { lity of } \\
\text { binary } \\
\text { data }\end{array}$ & $\begin{array}{l}\text { Unifor } \\
\mathrm{m} \\
\text { servic } \\
\mathrm{e} \quad \text { to } \\
\text { patient } \\
\mathrm{s} \text {, } \\
\text { feasibl } \\
\mathrm{e}, \\
\text { inexpe } \\
\text { nsive }\end{array}$ & $\begin{array}{l}\text { Overh } \\
\text { ead } \\
\text { due to } \\
\text { authen } \\
\text { ticatio } \\
\text { n of } \\
\text { users. } \\
\text { Micro- } \\
\text { contro } \\
\text { ller of } \\
\text { higher } \\
\text { config } \\
\text { uratio } \\
\text { n can } \\
\text { be } \\
\text { used. }\end{array}$ \\
\hline
\end{tabular}




\begin{tabular}{|c|c|c|c|c|c|}
\hline $\begin{array}{l}\text { Data } \\
\text { Mining } \\
\text { for the } \\
\text { Internet } \\
\text { of Things: } \\
\text { Literature } \\
\text { Review } \\
\text { and } \\
\text { Challenge } \\
\mathrm{s}[19]\end{array}$ & $\begin{array}{l}\text { Review } \\
\text { of } \\
\text { various } \\
\text { data } \\
\text { mining } \\
\text { techniq } \\
\text { ues and } \\
\text { its } \\
\text { applicat } \\
\text { ions is } \\
\text { perform } \\
\text { ed }\end{array}$ & ------- & $\begin{array}{l}3 \text { views } \\
\text { of data } \\
\text { mining } \\
--> \\
\text { knowle } \\
\text { dge, } \\
\text { techniq } \\
\text { ue, } \\
\text { applica } \\
\text { tion } \\
\text { view. }\end{array}$ & $\begin{array}{l}\text { Big } \\
\text { data, } \\
\text { data } \\
\text { minin } \\
\text { g are } \\
\text { hot } \\
\text { topics } \\
\text { to } \\
\text { discov } \\
\text { er } \\
\text { deep. }\end{array}$ & $\begin{array}{l}\text { Param } \\
\text { eter } \\
\text { optimi } \\
\text { zation } \\
\text { is not } \\
\text { consid } \\
\text { ered }\end{array}$ \\
\hline $\begin{array}{l}\text { Combinin } \\
\text { g KNN } \\
\text { Algorith } \\
\mathrm{m} \text { and } \\
\text { Other } \\
\text { Classifier } \\
\mathrm{s} \text { [20] }\end{array}$ & $\begin{array}{l}\text { KNN, } \\
\text { C4.5, } \\
\text { SVM } \\
\text { And } \\
\text { Naive } \\
\text { Bayes } \\
\text { Classifi } \\
\text { er(KNC } \\
\text { ) }\end{array}$ & $\begin{array}{l}20 \\
\text { UCI } \\
\text { Datase } \\
\text { ts }\end{array}$ & $\begin{array}{l}\text { Accura } \\
\text { cy for } \\
\text { classifi } \\
\text { cation }\end{array}$ & $\begin{array}{l}\text { Highe } \\
\mathrm{r} \\
\text { accura } \\
\text { cy }\end{array}$ & $\begin{array}{l}\text { Execut } \\
\text { ion } \\
\text { time } \\
\text { not } \\
\text { consid } \\
\text { ered }\end{array}$ \\
\hline $\begin{array}{l}\text { Intelligent } \\
\text { Urban } \\
\text { Traffic } \\
\text { Managem } \\
\text { ent } \\
\text { System } \\
\text { Based on } \\
\text { Cloud } \\
\text { Computin } \\
\text { g and } \\
\text { Internet } \\
\text { of } \\
\text { Things[3] }\end{array}$ & $\begin{array}{l}\text { Three } \\
\text { layers } \\
\text { of IOT } \\
\text { architec } \\
\text { ture } \\
\text { were } \\
\text { combin } \\
\text { ed with } \\
\text { SOA }\end{array}$ & '-------- & $\begin{array}{l}\text { Accura } \\
\text { cy, } \\
\text { Effecti } \\
\text { veness }\end{array}$ & $\begin{array}{l}\text { Specif } \\
\text { ic } \\
\text { applic } \\
\text { ations } \\
\text { were } \\
\text { realize } \\
\text { d such } \\
\text { as } \\
\text { intelli } \\
\text { gent } \\
\text { traffic } \\
\text { contro } \\
\text { l, } \\
\text { intelli } \\
\text { gent } \\
\text { vehicl } \\
\text { e } \\
\text { guidan } \\
\text { ce, } \\
\text { intelli } \\
\text { gent } \\
\text { accide } \\
\text { nt } \\
\text { monit } \\
\text { oring } \\
\text { etc. }\end{array}$ & $\begin{array}{l}\text { No } \\
\text { real- } \\
\text { time } \\
\text { data is } \\
\text { involv } \\
\text { ed } \\
\text { here }\end{array}$ \\
\hline $\begin{array}{l}\text { Internet } \\
\text { of things: } \\
\text { Vision, } \\
\text { applicatio } \\
\text { ns and } \\
\text { research } \\
\text { challenge } \\
\text { s[21] }\end{array}$ & $\begin{array}{l}\text { Review } \\
\text { of IOT } \\
\text { along } \\
\text { with the } \\
\text { challeng } \\
\text { es is } \\
\text { discusse } \\
\text { d. }\end{array}$ & -------- & ------- & $\begin{array}{l}\text { IOT } \\
\text { applic } \\
\text { ations } \\
\text { are } \\
\text { descri } \\
\text { bed } \\
\text { ensuri } \\
\text { ng its } \\
\text { efficie } \\
\text { nt use } \\
\text { in } \\
\text { future } \\
\text { work }\end{array}$ & $\begin{array}{l}\text { No } \\
\text { param } \\
\text { eter } \\
\text { enhan } \\
\text { cemen } \\
\mathrm{t} \\
\text { mecha } \\
\text { nism } \\
\text { is } \\
\text { consid } \\
\text { ered }\end{array}$ \\
\hline $\begin{array}{l}\text { Smartpho } \\
\text { ne-Based } \\
\text { Automati } \\
\text { c }\end{array}$ & $\begin{array}{l}\text { Viola } \\
\text { Jones } \\
\text { algorith } \\
\mathrm{m},\end{array}$ & $\begin{array}{l}\text { Ultras } \\
\text { ound } \\
\text { images } \\
\text { from }\end{array}$ & $\begin{array}{l}\text { Predicti } \\
\text { on } \\
\text { accurac } \\
\text { y }\end{array}$ & $\begin{array}{l}\text { Benefi } \\
\text { ts } \\
\text { rural } \\
\text { people }\end{array}$ & $\begin{array}{l}\text { Only } \\
\text { cyst } \\
\text { and } \\
\text { kidney }\end{array}$ \\
\hline
\end{tabular}

\begin{tabular}{|c|c|c|c|c|c|}
\hline $\begin{array}{l}\text { Abnormal } \\
\text { ity } \\
\text { Detection } \\
\text { of Kidney } \\
\text { in } \\
\text { Ultrasoun } \\
\text { d } \\
\text { Images[2 } \\
\text { 2] }\end{array}$ & $\begin{array}{l}\text { SVM, } \\
\text { Genetic } \\
\text { algorith } \\
\mathrm{m}\end{array}$ & $\begin{array}{l}\text { the } \\
\text { ultraso } \\
\text { und } \\
\text { scanne } \\
r\end{array}$ & & $\begin{array}{l}\text { can be } \\
\text { used } \\
\text { for } \\
\text { emerg } \\
\text { ency }\end{array}$ & $\begin{array}{l}\text { stone } \\
\text { is } \\
\text { consid } \\
\text { ered }\end{array}$ \\
\hline $\begin{array}{l}\text { Spatial } \\
\text { and } \\
\text { Temporal } \\
\text { Patterns } \\
\text { in Large- } \\
\text { Scale } \\
\text { Traffic } \\
\text { Speed } \\
\text { Prediction } \\
\text { [23] }\end{array}$ & $\begin{array}{l}\text { Unsuper } \\
\text { vised } \\
\text { methods } \\
\text { (k- } \\
\text { means, } \\
\text { self- } \\
\text { organizi } \\
\text { ng } \\
\text { maps, } \\
\text { principa } \\
\text { l } \\
\text { compon } \\
\text { ent } \\
\text { analysis } \\
\text { ) to find } \\
\text { out } \\
\text { global } \\
\text { trends }\end{array}$ & $\begin{array}{l}\text { Road } \\
\text { networ } \\
\mathrm{k} \text { from } \\
\text { Outra } \\
\mathrm{m} \\
\text { Park } \\
\text { to } \\
\text { Chang } \\
\text { i in } \\
\text { Singap } \\
\text { ore. }\end{array}$ & $\begin{array}{l}\text { Predicti } \\
\text { on } \\
\text { accurac } \\
\text { y } \\
\text { MSE }\end{array}$ & $\begin{array}{l}\text { Spatia } \\
1 \text { and } \\
\text { tempo } \\
\text { ral } \\
\text { trends } \\
\text { found } \\
\text { which } \\
\text { was } \\
\text { not } \\
\text { possib } \\
\text { le } \\
\text { throug } \\
\text { h the } \\
\text { use of } \\
\text { SVM }\end{array}$ & $\begin{array}{l}\text { Need } \\
\text { to } \\
\text { incorp } \\
\text { orate } \\
\text { these } \\
\text { found } \\
\text { pattern } \\
\mathrm{s} \text { into } \\
\text { route } \\
\text { guidin } \\
\text { g } \\
\text { algorit } \\
\text { hms }\end{array}$ \\
\hline $\begin{array}{l}\text { Improvin } \\
\mathrm{g} \text { Traffic } \\
\text { Prediction } \\
\text { with } \\
\text { Tweet } \\
\text { Semantics } \\
{[24]}\end{array}$ & $\begin{array}{l}\text { Correlat } \\
\text { ion } \\
\text { analysis } \\
\text { between } \\
\text { traffic } \\
\text { measure } \\
\text { ments } \\
\text { and a } \\
\text { number } \\
\text { of } \\
\text { tweets. } \\
\text { Later } \\
\text { optimiz } \\
\text { ation } \\
\text { framew } \\
\text { ork was } \\
\text { used. }\end{array}$ & $\begin{array}{l}\text { Traffic } \\
\text { and } \\
\text { data } \\
\text { from } \\
\text { Twitte } \\
r>> \\
\text { San } \\
\text { Franci } \\
\text { sco } \\
\text { Bay } \\
\text { area of } \\
\text { Califor } \\
\text { nia }\end{array}$ & $\begin{array}{l}\text { MAPE } \\
\text { and } \\
\text { RMSE }\end{array}$ & $\begin{array}{l}\text { Predic } \\
\text { tion } \\
\text { better } \\
\text { in } \\
\text { compa } \\
\text { rison } \\
\text { to the } \\
\text { auto- } \\
\text { correl } \\
\text { ation } \\
\text { model }\end{array}$ & $\begin{array}{l}\text { Spam } \\
\text { data } \\
\text { presen } \\
\text { ce, no } \\
\text { work } \\
\text { on } \\
\text { hetero } \\
\text { geneo } \\
\text { us } \\
\text { traffic. }\end{array}$ \\
\hline $\begin{array}{l}\text { Road } \\
\text { Traffic } \\
\text { Parameter } \\
\text { s } \\
\text { Prediction } \\
\text { In Urban } \\
\text { Traffic } \\
\text { Managem } \\
\text { ent } \\
\text { Systems } \\
\text { Using } \\
\text { Neural } \\
\text { Networks } \\
\text { [25] }\end{array}$ & $\begin{array}{l}\text { Neural } \\
\text { Networ } \\
\text { ks }\end{array}$ & ------- & $\begin{array}{l}\text { Accura } \\
\text { cy }\end{array}$ & $\begin{array}{l}\text { Only } \\
\text { for } \\
\text { short- } \\
\text { term } \\
\text { predic } \\
\text { tion }\end{array}$ & $\begin{array}{l}\text { The } \\
\text { better } \\
\text { predict } \\
\text { ion } \\
\text { model } \\
\text { is } \\
\text { neede } \\
\mathrm{d} \text { for } \\
\text { long- } \\
\text { term } \\
\text { predict } \\
\text { motio } \\
\mathrm{n} \text { of } \\
\text { traffic }\end{array}$ \\
\hline $\begin{array}{l}\text { Smart } \\
\text { video } \\
\text { surveillan } \\
\text { ce system } \\
\text { for } \\
\text { vehicle }\end{array}$ & $\begin{array}{l}\text { Image } \\
\text { Processi } \\
\text { ng-- } \\
>\text { Backg } \\
\text { round } \\
\text { Subtract }\end{array}$ & $\begin{array}{l}\text { Video } \\
\text { Databa } \\
\text { se }\end{array}$ & $\begin{array}{l}\text { False } \\
\text { Rejecti } \\
\text { on } \\
\text { Rate(F } \\
\text { RR), } \\
\text { False }\end{array}$ & $\begin{array}{l}\text { Predic } \\
\text { tion } \\
\text { accura } \\
\text { cy is } \\
\text { increa } \\
\text { sed by }\end{array}$ & $\begin{array}{l}\text { Camer } \\
\text { as not } \\
\text { for } \\
\text { night } \\
\text { vision, } \\
\text { situati }\end{array}$ \\
\hline
\end{tabular}




\begin{tabular}{|c|c|c|c|c|c|}
\hline $\begin{array}{l}\text { Detection } \\
\text { and traffic } \\
\text { flow } \\
\text { control[2 } \\
6]\end{array}$ & $\begin{array}{l}\text { ion } \\
\text { using } \\
\text { Thresho } \\
\text { ld } \\
\text { Adjusti } \\
\text { ng } \\
\text { process }\end{array}$ & & $\begin{array}{l}\text { Accept } \\
\text { ance } \\
\text { Rate(F } \\
\text { AR), } \\
\text { Total } \\
\text { Succes } \\
\text { s } \\
\text { Rate(T } \\
\text { SR) }\end{array}$ & $\begin{array}{l}\text { the } \\
\text { use of } \\
\text { video } \\
\text { surveil } \\
\text { lance }\end{array}$ & $\begin{array}{l}\text { ons to } \\
\text { suspec } \\
\mathrm{t} \\
\text { danger } \\
\text { not } \\
\text { covere } \\
\text { d. }\end{array}$ \\
\hline $\begin{array}{l}\text { Utilizing } \\
\text { Real- } \\
\text { World } \\
\text { Transport } \\
\text { ation Data } \\
\text { for } \\
\text { Accurate } \\
\text { Traffic } \\
\text { Prediction } \\
\text { [27] }\end{array}$ & $\begin{array}{l}\text { H- } \\
\text { ARIMA } \\
+(\text { Hybri } \\
\text { d model } \\
\text { of HAM } \\
\text { and } \\
\text { ARIMA } \\
\text { ) }\end{array}$ & $\begin{array}{l}\text { Los } \\
\text { Angel } \\
\text { es } \\
\text { Count } \\
\text { y } \\
\text { Transp } \\
\text { ort } \\
\text { Netwo } \\
\text { rk }\end{array}$ & $\begin{array}{l}\text { MAPE } \\
\text { and } \\
\text { RMSE }\end{array}$ & $\begin{array}{l}\text { Short } \\
\text { term } \\
\text { and } \\
\text { Long } \\
\text { term } \\
\text { predic } \\
\text { tion } \\
\text { accura } \\
\text { cy } \\
\text { better } \\
\text { than } \\
\text { ARIM } \\
\text { A, ES, } \\
\text { NNet }\end{array}$ & 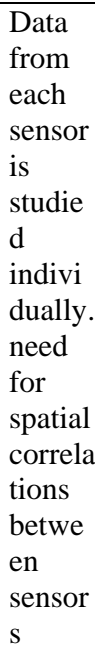 \\
\hline $\begin{array}{l}\text { A } \\
\text { Compreh } \\
\text { ensive } \\
\text { Review } \\
\text { on Traffic } \\
\text { Prediction } \\
\text { for } \\
\text { Intelligent } \\
\text { Transport } \\
\text { System[2 } \\
\text { 8] }\end{array}$ & $\begin{array}{l}\text { Review } \\
\text { of } \\
\text { techniq } \\
\text { ues used } \\
\text { in ITS } \\
\text { is } \\
\text { consider } \\
\text { ed like } \\
\text { NN, } \\
\text { fuzzy, } \\
\text { SVM, } \\
\text { Bayesia } \\
\text { n etc }\end{array}$ & $\begin{array}{l}\text { PMS, } \\
\text { TMC, } \\
\text { MIDA } \\
\text { S, } \\
\text { Bing } \\
\text { data, } \\
\text { Twitte } \\
\text { r Data. }\end{array}$ & $\begin{array}{l}\text { RMSE, } \\
\text { MAPE, } \\
\text { MRE, } \\
\text { VAPE, } \\
\text { EC etc }\end{array}$ & $\begin{array}{l}\text { Techn } \\
\text { iques } \\
\text { are } \\
\text { given } \\
\text { that } \\
\text { can be } \\
\text { enhan } \\
\text { ced in } \\
\text { future } \\
\text { work } \\
\text { for } \\
\text { predic } \\
\text { tion } \\
\text { accura } \\
\text { cy }\end{array}$ & $\begin{array}{l}\text { Lack } \\
\text { of use } \\
\text { of } \\
\text { deep } \\
\text { learnin } \\
\text { g } \\
\text { techni } \\
\text { ques, } \\
\text { dataset } \\
\text { s } \\
\text { exclud } \\
\text { ed } \\
\text { param } \\
\text { eters } \\
\text { such } \\
\text { as } \\
\text { humid } \\
\text { ity, } \\
\text { holida } \\
\text { ys etc. }\end{array}$ \\
\hline $\begin{array}{l}\text { An } \\
\text { Aggregati } \\
\text { on } \\
\text { Approach } \\
\text { to Short- } \\
\text { Term } \\
\text { Traffic } \\
\text { Flow } \\
\text { Prediction } \\
{[29]}\end{array}$ & $\begin{array}{l}\text { Integrati } \\
\text { on of } \\
\text { MA, } \\
\text { ES, and } \\
\text { ARIMA } \\
\text { using } \\
\text { NN }\end{array}$ & $\begin{array}{l}\text { Nation } \\
\text { al } \\
\text { Highw } \\
\text { ay } \\
107, \\
\text { Guang } \\
\text { zhou, } \\
\text { Guang } \\
\text { dong, } \\
\text { China }\end{array}$ & $\begin{array}{l}\text { RMSE, } \\
\text { PAE, } \\
\text { and } \\
\text { MAPE }\end{array}$ & $\begin{array}{l}\text { Accur } \\
\text { acy is } \\
\text { high }\end{array}$ & $\begin{array}{l}\text { The } \\
\text { situati } \\
\text { on } \\
\text { involv } \\
\text { ing } \\
\text { multip } \\
\text { le } \\
\text { detect } \\
\text { ors is } \\
\text { missin } \\
\mathrm{g}\end{array}$ \\
\hline $\begin{array}{l}\text { Traffic } \\
\text { big data } \\
\text { prediction } \\
\text { and } \\
\text { visualizati } \\
\text { on using }\end{array}$ & FIMT & $\begin{array}{l}\text { Depart } \\
\text { ment } \\
\text { of } \\
\text { Transp } \\
\text { ort UK }\end{array}$ & $\begin{array}{l}\text { Predicti } \\
\text { on } \\
\text { accurac } \\
\text { y } \\
\text { through } \\
\text { MAE, }\end{array}$ & $\begin{array}{l}\text { Accur } \\
\text { acy is } \\
\text { high } \\
\text { and } \\
\text { visuali } \\
\text { zation }\end{array}$ & $\begin{array}{l}\text { Means } \\
\text { square } \\
\text { error } \\
\text { can be } \\
\text { further } \\
\text { reduce }\end{array}$ \\
\hline
\end{tabular}

\begin{tabular}{|c|c|c|c|c|c|}
\hline $\begin{array}{l}\text { Fast } \\
\text { Increment } \\
\text { al Model } \\
\text { Trees- } \\
\text { Drift } \\
\text { Detection } \\
\text { (FIMT- } \\
\text { DD)[30] }\end{array}$ & & & $\begin{array}{l}\text { RMSE, } \\
\text { and } \\
\text { SMAP } \\
\text { E }\end{array}$ & $\begin{array}{l}\text { of } \\
\text { traffic } \\
\text { presen } \\
\text { ted for } \\
\text { better } \\
\text { unders } \\
\text { tandin } \\
\mathrm{g}\end{array}$ & d. \\
\hline $\begin{array}{l}\text { Traffic } \\
\text { Flow } \\
\text { Forecasti } \\
\text { ng Using } \\
\text { a } \\
\text { Spatial- } \\
\text { temporal } \\
\text { Bayesian } \\
\text { Network } \\
\text { Predictor[ } \\
\text { 31] }\end{array}$ & $\begin{array}{l}\text { Bayesia } \\
\mathrm{n} \\
\text { Networ } \\
\mathrm{k}\end{array}$ & -------- & $\begin{array}{l}\text { Accura } \\
\text { cy } \\
\text { through } \\
\text { MMSE }\end{array}$ & $\begin{array}{l}\text { Predic } \\
\text { tion } \\
\text { accura } \\
\text { cy is } \\
\text { impro } \\
\text { ved } \\
\text { since } \\
\text { pre- } \\
\text { proces } \\
\text { sing } \\
\text { reduce } \\
\text { s the } \\
\text { impact } \\
\text { of the } \\
\text { error }\end{array}$ & $\begin{array}{l}\text { No } \\
\text { real- } \\
\text { time } \\
\text { dataset } \\
\text { is } \\
\text { consid } \\
\text { ered }\end{array}$ \\
\hline
\end{tabular}

Table 2: Comparison of Proposed and Existing Technique with different $K$ values

\begin{tabular}{|l|l|l|l|}
\hline Lane & K & $\begin{array}{l}\text { Without } \\
\text { Euclidean } \\
\text { Distance }\end{array}$ & $\begin{array}{l}\text { With Euclidean } \\
\text { Distance }\end{array}$ \\
\hline 1 & Error & Error \\
\hline 1 & 2 & 6.67 & 5 \\
\hline 1 & 3 & 19.43 & 1.73 \\
\hline 1 & 4 & 36.95 & 2.34 \\
\hline 2 & 5 & 60.47 & 2.79 \\
\hline 2 & 2 & 6.54 & 1.55 \\
\hline 2 & 3 & 18.54 & 5.72 \\
\hline 2 & 4 & 36.87 & 11.09 \\
\hline 3 & 5 & 60.55 & 14.78 \\
\hline 3 & 2 & 6.37 & 1.01 \\
\hline 3 & 3 & 18.81 & 5.85 \\
\hline 3 & 4 & 35.71 & 12.34 \\
\hline & 5 & 57.55 & 15.71 \\
\hline
\end{tabular}




\section{RESULT}

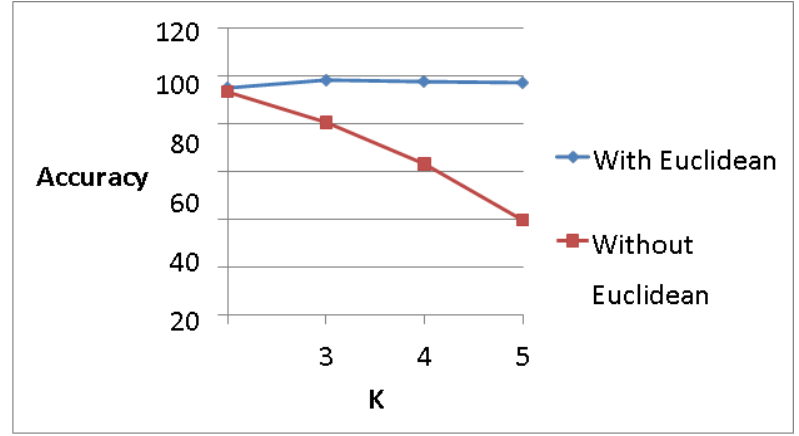

Fig 1: First Comparative Analysis of proposed and existing technique

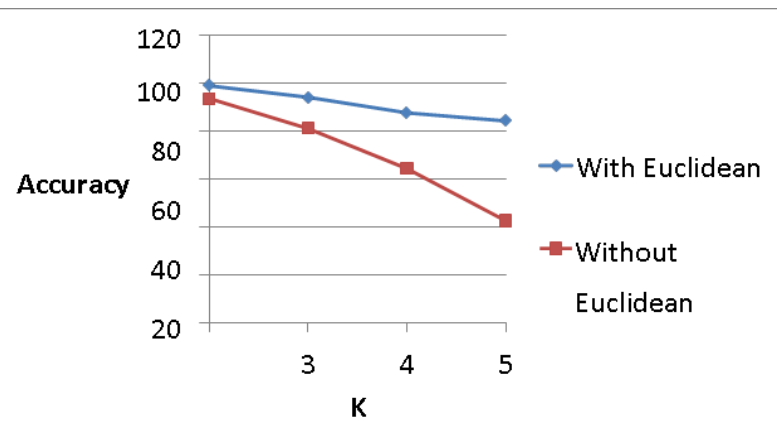

Fig 3: Second Comparative Analysis of proposed and existing technique

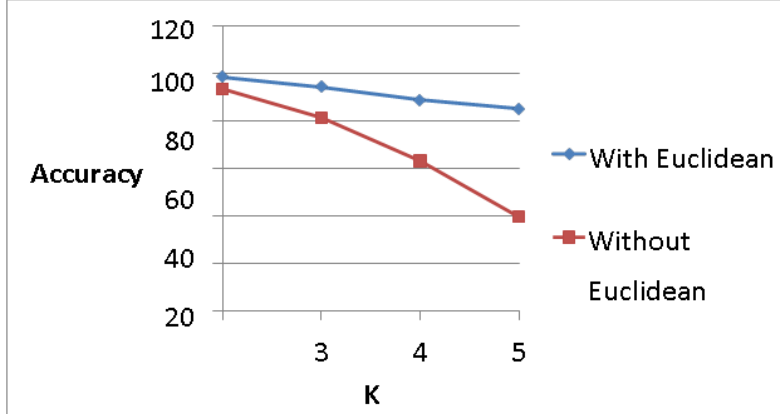

Fig 2: Third Comparative Analysis of proposed and existing technique

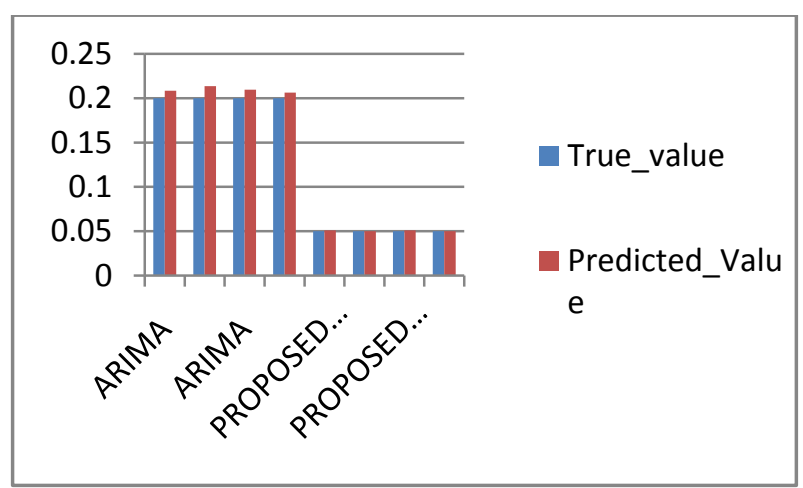

Fig 4: Final Comparative Analysis of proposed and existing technique

Table 3: Examination of existing technique without KE-ARIMA distance

\begin{tabular}{|l|l|l|l|l|}
\hline FUNCTIONS & \multicolumn{2}{|l|}{ ARIMA } & KE-ARIMA & \multicolumn{1}{l|}{} \\
\cline { 2 - 5 } & True Value & Predictive Value & True Value & Predictive Value \\
\hline Mean square error & 5.6987 & 5.77981 & 5.6987 & 5.70263 \\
\cline { 5 - 5 } & & & & 5.76168 \\
\cline { 3 - 5 } & & 5.9835 & & \\
\hline
\end{tabular}

\section{CONCLUSION \& FUTURE SCOPE}

In this thesis, we proposed a new prediction approach by combining $\mathrm{K}$ Nearest Neighbor techniques and Euclidean Distance with traditional time series prediction ARIMA modeling.

The new approach has been tested to predict the network traffic. We analyzed the network traffic data and extracted useful data from the dataset. We explored the effectiveness and usefulness of prediction techniques by applying KNN and Euclidean Distance to observe new point in the network. We proposed a KNN and ED based traffic prediction method. We applied the KNN and ED based ARIMA model to predict the network traffic. Another contribution of this research project is the illustration how data mining techniques may be used to help solve practical real-world problems. In the most recent decade, the examination and forecast of system activity has turned into a subject of persistent research in different subfields of PC systems. Countless number of scientists have been executed a successful organize movement calculation for the examination and the expectation of system movement. The proposed method utilizes hybrid approach of KNN and Euclidean distance to achieve prediction accuracy, prediction accuracy can further be enhanced by reduction in MSE and
RMSE. Proposed hybrid approach achieves the same and hence is optimal in nature.

Proposed system ARIMA with KE-ARIMA distance produces better result as compared to existing techniques without KEARIMA distance. In future, SVM can be used for better prediction of traffic.

\section{ACKNOWLEDGMENTS}

Firstly I whole heartedly thank Almighty for enlightening new paths whenever I faced any problems. Also many people have shared their time and expertise to help me accomplish my goal. The help and cooperation extended by Head of department (CSE), I.K.G Punjab Technical University Main Campus, Kapurthala is fully acknowledged. I would like to take this opportunity to express my deepest sense of gratitude and profound feeling of admiration to my dissertation supervisor, Mr. Rajdeep Singh, for his guidance, motivation, enthusiasm and immense knowledge. His around-the-clock instant response to my countless queries has been invaluable and extremely motivational. I would also like to thank everyone who has knowingly \&amp; unknowingly helped me throughout my dissertation anytime. I would also like to thank the authors of all those books and papers which I have 
referred during my work as well as for preparing the report. At last I express my sincere gratitude to the I.K.G Technical University Main Campus, Kapurthala for giving me the opportunity to work on the dissertation during my final year of Masters.

\section{REFERENCES}

[1] W. Min and L. Wynter, "Real-time road traffic prediction with spatio-temporal correlations," Transp. Res. Part C, vol. 19, no. 4, pp. 606-616, 2011.

[2] S. V. Kumar and L. Vanajakshi, "Short-term traffic flow prediction using seasonal ARIMA model with limited input data," Eur. Transp. Res. Rev., vol. 7, no. 3, pp. 1-9, 2015.

[3] X. Yu, "Intelligent Urban Traffic Management System Based on Cloud Computing and Internet of Things," pp. 2169-2172, 2012.

[4] X. Pang, C. Wang, and G. Huang, "A Short-Term Traffic Flow Forecasting Method Based on a Three-Layer KNearest Neighbor Non-Parametric Regression Algorithm," no. July, pp. 200-206, 2016.

[5] O. U. Chinyere, O. O. Francisca, and O. E. Amano, "D ESIGN AND S IMULATION OF AN I NTELLIGENT T RAFFIC," vol. 1, no. 5, pp. 47-57, 2011.

[6] C. Xiao-feng, S. Zhong-ke, and Z. Kai, "Research on an Intelligent Traffic Signal Controller," pp. 884-887, 2003.

[7] L. Kdqj, "An Intelligent Traffic Information Service System based on Agent and GIS-T," 2010.

[8] B. Singh and A. Gupta, "Recent trends in intelligent transportation systems : a review," vol. 9, no. 2, pp. 3034, 2015.

[9] H. O. Al-sakran, "Intelligent Traffic Information System Based on Integration of Internet of Things and Agent Technology," vol. 6, no. 2, pp. 37-43, 2015.

[10] T. Osman, S. S. Psyche, J. M. S. Ferdous, and H. U. Zaman, "Intelligent Traffic Management System for Cross Section of Roads Using Computer Vision," 2017.

[11] Y. Wang and H. Qi, "Research of Intelligent Transportation System Based on the Internet of Things Frame," vol. 2012, no. July, pp. 160-166, 2012.

[12] M. Kim and S. Chang, "," vol. 62, no. 3, pp. 226-234, 2016.

[13]C. U. Scenarios and R. Architecture, "The advantages of IoT and Cloud applied to Smart Cities," pp. 325-332, 2015.

[14] S. Vasantha Kumar and Lelitha Vanajakshi, "Short-term traffic flow prediction using seasonal ARIMA model with limited input data," pp. 1-9, 2016.

[15] A. Mathew, F. A. S. A, H. N. Pooja, and A. Verma, "Smart Disease Surveillance Based on Internet of Things ( IoT )," vol. 4, no. 5, pp. 180-183, 2015.

[16] B. D. Thomas, R. Mcpherson, G. Paul, and J. Irvine, "Consumption of Wi-Fi for IoT Devices," no. September, pp. 92-100, 2016.

[17] C. Perera, D. S. Talagala, C. H. Liu, S. Member, and J. C. Estrella, "Energy-Efficient Location and ActivityAware On-Demand Mobile Distributed Sensing Platform for Sensing as a Service in IoT Clouds," pp. 1-11, 2016.

[18] J. Mohammed, C.-H. Lung, A. Ocneanu, A. Thakral, C. Jones, and A. Adler, "Internet of Things: Remote Patient Monitoring Using Web Services and Cloud Computing," in 2014 IEEE International Conference on Internet of Things(iThings), and IEEE Green Computing and Communications (GreenCom) and IEEE Cyber, Physical and Social Computing (CPSCom), 2014, pp. 256-263.

[19] F. Chen, P. Deng, J. Wan, D. Zhang, A. V Vasilakos, and $X$. Rong, "Data Mining for the Internet of Things: Literature Review and Challenges," vol. 2015, no. i, 2015.

[20] Z. Zhou, C. Du, L. Shu, G. Hancke, J. Niu, and H. Ning, "Combining KNN Algorithm and Other Classifiers ," in 2010 IEEE International Conference on Cognitive Informatics, 2010,pp.800-805.

[21] D. Miorandi, S. Sicari, F. De Pellegrini, and I. Chlamtac, "Ad Hoc Networks Internet of things: Vision, applications and research challenges," Ad Hoc Networks, vol. 10, no. 7, pp. 1497-1516, 2012.

[22] P. Vaish, R. Bharath, P. Rajalakshmi, and U. B. Desai, "Smartphone Based Automatic Abnormality Detection of Kidney in Ultrasound Images," 2016.

[23] M. T. Asif, J. Dauwels, C. Y. Goh, A. Oran, E. Fathi, M. $\mathrm{Xu}$, M. M. Dhanya, N. Mitrovic, and P. Jaillet, "Spatial and Temporal Patterns in Large-Scale Traffic Speed Prediction."

[24] J. He, W. Shen, P. Divakaruni, L. Wynter, and R. Lawrence, "Improving Traffic Prediction with Tweet Semantics," pp. 1387-1393.

[25] P. Transportu and T. Pamuła, "ROAD TRAFFIC PARAMETERS PREDICTION IN URBAN TRAFFIC MANAGEMENT SYSTEMS USING NEURAL NETWORKS," Transport, vol. 6, no. 3, pp. 1-6, 2011.

[26] A. A. Shafie, M. H. Ali, F. Hafiz, and R. M. Ali, "SMART VIDEO SURVEILLANCE SYSTEM FOR VEHICLE DETECTION AND TRAFFIC FLOW CONTROL," vol. 6, no. 4, pp. 469-480, 2011.

[27] B. Pan, U. Demiryurek, and C. Shahabi, "Utilizing RealWorld Transportation Data for Accurate Traffic Prediction."

[28] S. Suhas, V. K. V, M. Katti, A. P. B. V, and C. Naveena, "A Comprehensive Review on Traffic Prediction for Intelligent Transport System," 2017.

[29] M. Tan, S. C. Wong, J. Xu, Z. Guan, and P. Zhang, “An Aggregation Approach to Short-Term Traffic Flow Prediction," vol. 10, no. 1, pp. 60-69, 2009.

[30] A. Wibisono, W. Jatmiko, H. A. Wisesa, B. Hardjono, and P. Mursanto, "Knowledge-Based Systems Traffic big data prediction and visualization using Fast Incremental Model Trees-Drift Detection ( FIMT-DD )," KnowledgeBased Syst., vol. 93, pp. 33-46, 2016.

[31] S. Sun, C. Zhang, and Y. Zhang, "Traffic Flow Forecasting Using a Spatio-temporal Bayesian Network Predictor," pp. 273-278, 2005. 